\title{
A CASE OF CLINICAL JUVENILE AMAUROTIC IDIOCY WITH THE HISTOLOGICAL PICTURE OF ALZHEIMER'S DISEASE
}

\author{
BY \\ Aa. CHRISTIE LÖKEN and K. CYVIN

\begin{abstract}
From the Neuropathological Laboratory, the Rikshospitalet, Oslo, and the Reitgjerdet Mental Hospital,
\end{abstract} \\ Trondheim, Norway
}

The clinical picture of juvenile amaurotic idiocy was originally described in Norway by Stengel in 1826. He observed four siblings with normal development up to the age of 6 years when amaurosis and subsequent mental disorders developed. From the age of 14 years convulsive seizures were noted. The disease ran a progressive course and the patients died at the age of about 20 years. Vogt in Germany in 1905 reported some similar cases and Spielmeyer in 1905 described a typical histopathological picture with globular swelling of the ganglion cells, and balloon-like swellings of the dendrites, due to lipoid deposits in the cytoplasm. In Sweden the disease seems to be relatively frequent. Sjögren (1931) collected 120 cases from 56 families. He examined 4,500 individuals and found a recessive monohybrid heredity. Sjövall and Ericsson (1933) examined biopsy and necropsy specimens from the Swedish cases and described lipoid storage in the cytoplasm of the nerve cells, especially in the cerebral cortex, and also in the Purkinje cells and in the neostriatum. They also pointed out an unusual macroglial reaction with lipoidosis of the glial cells. They found the picture not unlike that seen in normal senescence.

Josephy (1936) advised caution in basing the diagnosis on the clinical picture alone in the absence of a typical histological picture. Sjögren, however, held the opinion that a definite diagnosis could be based on the clinical picture alone, because this was exactly the same from case to case. He emphasized that the disease usually starts at the age of 5 to 8 years with a progressive decrease of vision, leading to complete amaurosis after one or two years. The papillae in the earlier stages are brownishyellow, later pale yellow or white, often with concentric zones. The retinal vessels, especially the arteries, are narrow, and atrophic areas occur in the eye ground. The pigmentations are granular, increasing toward the periphery. In the final stages a bilateral posterior cataract may develop, and the pupillary reflex is abolished. Increasing dementi. is a constant feature with disorders of the different intellectual spheres including understanding, memory, and judgment. There may be emotional lability with seizures of weeping and unmotivated laughter. The behaviour varies between laziness and restlessness but the patient is often able to obey orders. In a more advanced stage postural anomalies appear, with general weakness, kyphosis, genu valgum, and pes planus. The gait becomes unsteady and shuffling, and the patient may move for a time in one spot before starting to walk with the feet apart and dragging on the ground. There is a slow intention tremor involving the entire body when the patient starts walking (démarche trepidante). The speech becomes slurred, stuttering, and monotonous, and there is paucity of words and agrammatism. Facial mimicry is lost. The hearing is good; the reflexes are normal or increased. An oily appearance of the face and acrocyanosis indicate disturbed vegetative functions. Epileptic fits may sometimes be observed early, but usua!ly not until the blindness has become manifest. In the later stages contractures, rigidity, fibrillary muscular tremor, and decubitus follow, and the patients die from intercurrent diseases.

In Norway juvenile amaurotic idiocy is rare. By questioning all mental hospitals and blind schools 10 cases have been found, including the patient reported here, and his brother.

\section{Case Report}

The patient was admitted to Reitgjerdet Mental Hospital on October 6, 1945, at the age of 14 years. His parents were first cousins. There was no history of mental disease in the family as far back as his greatgreat-grandparents with the exception of the patient's older brother, who apparently had suffered from the same disease and died from an intercurrent disease at the age of 13 years. One sister died of pneumonia at the age of 15. Another brother, who was more than 20 years old, was alive and healthy. 
The patient developed normally up to the age of 6 , when impairment of vision and retarded mental development were noted. He attended school for one year but then had to be taken away because he was no longer able to read. He had by then learnt how to spell and put words together, but this was soon forgotten. He stayed at home on the farm with his father, and at times he was excited and difficult to handle. At the age of 13 years a convulsive seizure was observed, and several seizures occurred at varying intervals during the following years. His speech became less distinct, and a tendency to perseveration was noted. On admission to the hospital he was disoriented as to time, place, and situation. He knew his own name, gave short answers to simple questions, but was deeply demented. He was anxious when examined, resistive, and emotionally labile. Communication with the patient was difficult; sometimes he behaved calmly, but at other times he was restless and crying. He used to hide his head under the blankets, and had to be helped and nursed.

He looked well nourished. Acrocyanosis and marked dermographism were noted. Temperature and pulse rate were normal. His sleep was normal. The blood pressure was 100-125/70-90 mm. Hg. Blood examinations showed slight lymphocytosis, later marked eosinophilia $(25 \%$ eosinophils) and leucocytosis $(12,000$ leucocytes). The sedimentation rate varied between 20 and $32 \mathrm{~mm}$. There was no evidence of disease of the internal organs. Ssrological examinations of blood and cerebrospinal fluid gave normal findings. Cholesterol values were not determined because of technical difficulties. The electrocardiogram was normal. Ophthalmological examination showed marked mydriasis, and a sluggish pupillary reflex. He was able to distinguish

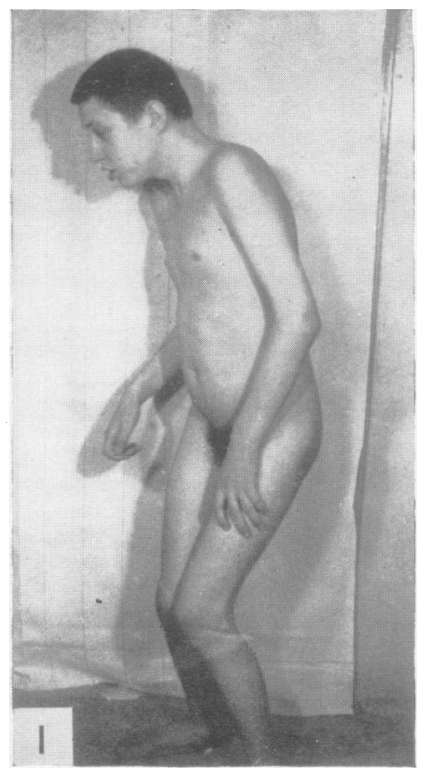

FIG. 1.-The patient. large objects at a short distance. The papillae were pale, distinctly marginated, surrounded by a yellowish, waxylooking zone. The arteries were narrow, and along them small granulated pigmentations without dendrites were observed. The maculae were not visible, and there was no cherry red spot.

Neurological examination disclosed slightly disturbed coordination, but no definite ataxia. Tactile sensation was impaired all over the body. The speech was slurred and difficult to understand, but there was no definite dysarthria. He kept his head and shoulders bent forward, and his knees were flexed (Fig. 1). The gait was unsteady. The reflexes of the upper and lower extremities were exaggerated, especially the patellar reflexes. The abdominal reflexes were present, and also the plantar reflexes. During his stay in hospital he had a few epileptic fits.

He became more and more helpless, demented, and untidy. Finally he was entirely confined to bed and died of bronchopneumonia on April 5, 1948.

Necropsy.-Necropsy showed no gross pathological changes except pneumonia. Microscopical examination of the liver showed some lipoid storage.

The Brain.-When examined the brain had been stored in formalin for nearly four years. The total weight was $850 \mathrm{~g}$. The cerebral hemispheres appeared evenly atrophied, while the cerebellum seemed to be of normal size. The cerebral gyri were narrow. and the sulci widened. The lateral ventricles were enlarged. The cortical surface appeared smooth, and on the cut surface the atrophy was mainly restricted to the white matter. Blocks from representative parts of the brain, cerebellum, and brain-stem were embedded in paraffin, stained with haematoxylin and eosin, thionin, Holzer glial fibre method, Bodian's silver method, and Loyez's method for myelin. Supplementary blocks were frozen and sections stained with Sudan III.

Microscopical Examination.-The leptomeninges and the vessels showed no pathological changes. The cortical pattern was quite well preserved, while there was a moderate and diffuse loss of nerve cells without preference for any special layer. The neurons were slightly irregular and their cytoplasm was shrunken, while the nuclei looked normal. The Nissl granules were mostly well stained. In some of the cells there was a moderate amount of sudanophil material, but no balloon-like swelling of the cells was noted in any part of the brain. The various areas examined all showed similar pictures, but certain parts such as the hippocampal formation were more severely involved. Neuronal changes of the same kind were also observed in the basal ganglia, striatum. pallidum, and thalamic nuclei. The cells were rather small and irregular. In the hypothalamic nuclei and the nuclei of the brainstem the neurons showed similar changes. The cerebellum was apparently intact. The Purkinje cells were nicely pear shaped, and there was no swelling of their dendrites. The dentate and the roof nuclei were normal. The glial elements were increased in number. There were numerous small elongated cells with irregular nuclei, resembling microglial cells, and also a great number of large astrocytes. In thionin sections, rounded, pale blue patches were scattered throughout the cortex, especially in the outer three layers. Holzer staining showed that these patches were made up partly by glial fibrils, and partly by a more densely staining ground substance (Fig. 2). With silver staining some of the patches were impregnated, and gave the picture of senile plaques (Figs. 3-4). Some of the microglial cells 

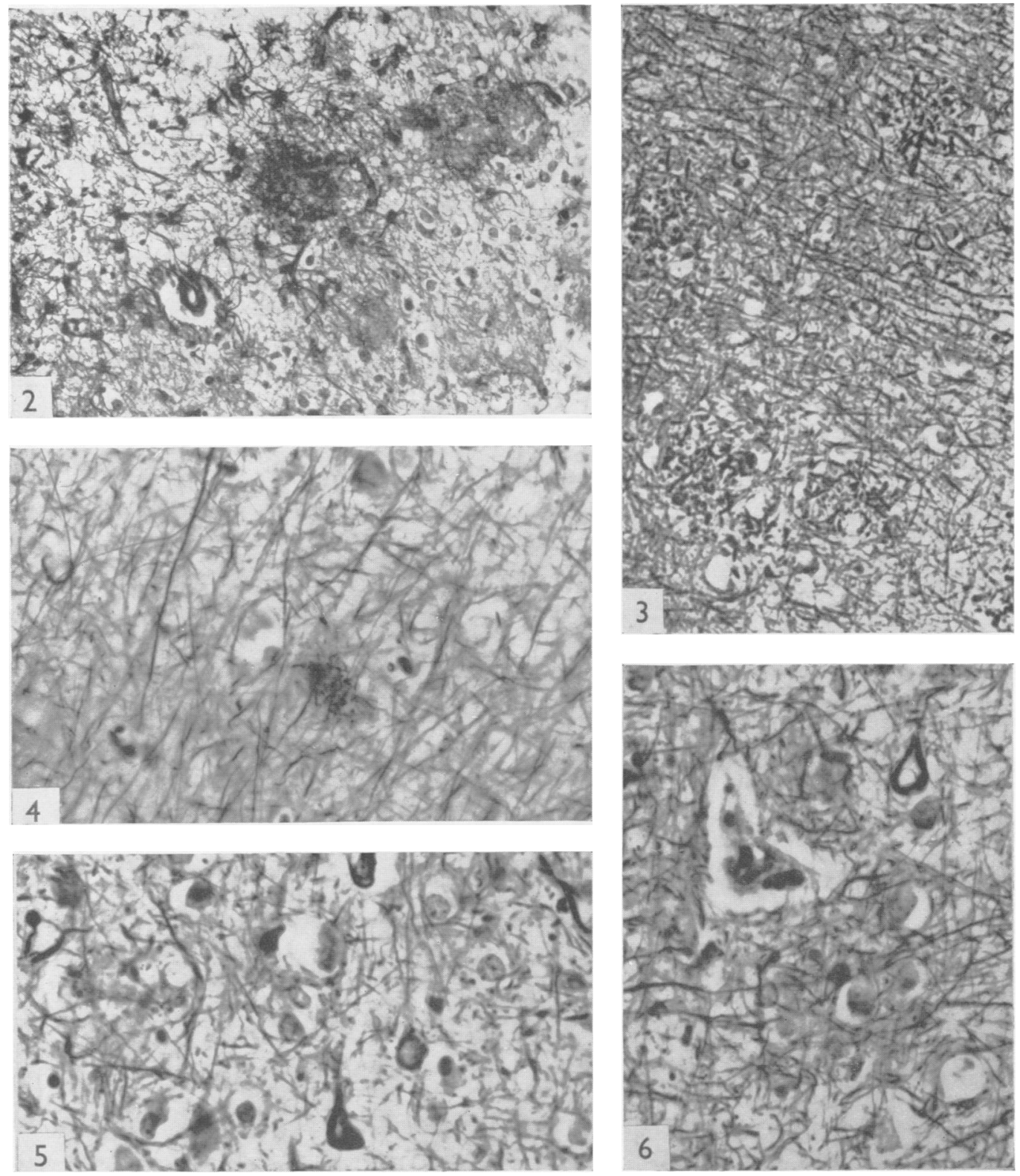

Fig. 2.-Dark-staining patches in the cortex partly consisting of glial fibres and partly due to a heavier staining of the background. Holzer. $\times 200$.

Figs. 3 and 4.-Senile plaques in the cortex. Bodian's silver method. $\times 200$ and $\times 400$.

Figs. 5 and 6.-Alzheimer's fibrils in cortical neurons. Bodian's silver method. $\times 400$.

contained sudanophil granules. Compound granular cells were few and only seen perivascularly in the white matter. There was a moderate loss of myelin in the white matter, and an increase of fibrillary glia. In the globus pallidus a few mulberry-shaped, iron-containing concretions were present in the perivascular spaces, and some of the arteries also contained iron deposits in their walls.

With Bodian's silver impregnation a great number of curved, thick intraneuronal fibres of the Alzheimer type 
were seen throughout the cerebral cortex (Figs. 5,6) but neither Alzheimer fibrils nor senile plaques were seen in the subcortical grey matter or cerebellum.

\section{Discussion}

The clinical picture in the present case was almost identical with that of juvenile amaurotic idiocy described by Sjögren. Of other possible diagnoses a syphilitic disease was considered, viz., either a juvenile general paralysis or a congenital form of neurosyphilis. These diseases may also be combined with papillary atrophy and retinal pigmentation. The papillae, however, are usually less distinctly marginated and the pigmentations darker. There may be posture anomalies, although spastic paretic contractures will usually predominate, with extra-pyramidal symptoms being less distinct. Serological tests were all negative.

In view of the rapid progression of the disease a diagnosis of degeneratio pigmentosa retinae with mental defects did not seem justified. The lack of pressure symptoms did not indicate hydrocephalus as the cause of the symptoms. Familial diffuse sclerosis of the Pelizaeus Merzbacher type, besides amaurosis and epilepsy, always includes spastic ataxic paralyses and spastic contractures. The cerebellar heredoataxia of Pierre Marie shows similar walking disturbances, but here cerebellar symptoms will always predominate. The lack of the cherry red spot and the relatively slow course made us reject also the congenital form of amaurotic idiocy.

The histological picture, however, was quite different from what was expected according to the clinical findings. Neuronal changes typical of amaurotic idiocy were not present, although some lipoid storage was observed. This, however, might be a result of any degenerative process, and may even be seen in senescence.

The general impression was indicative of a slowly progressing degenerative disease, primarily affecting the cerebral cortex, but also involving the nerve cells of the basal ganglia and the brain-stem. The absence of similar changes in the cerebellum was rather surprising. The moderate demyelinization was considered secondary to the neuronal disease. The presence of Alzheimer fibrils and the number of senile plaques throughout the cortex are unusual at this age.

A somewhat similar case has been described by Malamud and Lowenberg (1929) A man died at the age of 24 years from a disease which apparently had started very early following an attack of scarlet fever. His development was retarded, and when he was 14 mental deterioration became apparent. Later, both mental and physical symptoms pro- gressed. The authors described both Alzheimer fibrils and senile plaques in the cerebral cortex, but none in the cerebellum. They concluded that the Alzheimer symptom complex could not be confined to any particular age, and also that several aetiological factors are capable of producing similar pictures.

Lüers (1947) described a juvenile form of Alzheimer disease in a brother and a sister. The clinical diagnosis was multiple sclerosis. The symptoms started at the ages of 26 and 43 years, respectively. Lüers referred to a paper by von Braunmühl who found that the earlier Alzheimer's disease starts, the more atypical is the course.

Hallervorden (1935) reported Alzheimer changes in post-encephalitic brains in a total of 35 cases described by himself and others, the age of the patients varying between 11 and 60 years. He did not observe any senile plaques. He was of the opinion that fibrillary changes occurred only in chronic diseases, and were possibly due to colloidchemical processes leading to degeneration.

Meyer (1949) found a combined systemic disease in two sisters, 7 and 4 years old, with clinical cerebral palsy and idiocy. He found senile plaques. in the dentate hilum, but no fibrillary changes.

Jervis (1952) reported the case of an 11-year-old child with mental deterioration from birth, displaying a cherry red spot as in congenital and infantile forms of amaurotic idiocy. There were senile plaques in the cerebellum, and no real ballooning or swelling of cells and dendrites.

Van Bogaert (1952) reported on a family with tardive dementia combined with extrapyramidal features, which displayed histologically the picture of amaurotic idiocy although the lipoid storage was very moderate and the cell forms not quite typical. He also referred to a communication by Hallervorden who had seen a case of late lipoidosis combined with Alzheimer's fibrillary changes.

As to amaurotic idiocy, a congenital, an infantile, a late infantile, a juvenile, and late forms have been described, the last by Kufs (1931). Histological lipoidosis may consequently be seen at any age. On the other hand Alzheimer's fibrillary changes and senile plaques have been described in young subjects displaying a number of different clinical pictures.

The amaurotic idiocy has a somewhat different clinical symptomatology in its different age groups, the cherry red spot belonging to the youngest age group, the visual disturbances being more or less absent in the late forms, and the progression slower in the older groups. Perhaps the histological picture is even less pronounced in these late cases. 
Alzheimer's fibrillary changes have been found as an associated feature in amaurotic idiocy, and they have been described in subjects of different ages including children.

In the present case the clinical course was that of a classical juvenile amaurotic idiocy, while the histological pictures were suggestive of early Alzheimer disease. It is obvious that a slowly progressing degenerating disease caused the symptoms, but the question of whether the disease should be considered amaurotic idiocy or a juvenile form of Alzheimer's disease is left open.

\section{Summary}

A case of clinical juvenile amaurotic idiocy is reported. The gross and microscopical pictures of the brain are described. Histological sections failed to disclose swollen nerve cells as in lipoidosis, and a diffuse cortical atrophy with Alzheimer's fibrillary changes in the ganglion cells and numerous senile plaques were found.

\section{REFERENCES}

Bogaert, L. van (1952). Dtsch. Z. Nervenheilk., 168, 267.

Hallervorden, J. (1935). Ibid., 136, 68.

Jervis, G. A. (1952). J. Neuropath., 11, 4.

Josephy, H. (1936). ed. Bumke, O., and Foerster, O. Handbuch der Neurologie, vol. 16, p. 394.' Springer, Berlin.

Kufs, H. (1931). Z. ges. Neurol. Psychiat., 137, 432.

Lüers, Th. (1947). Arrh. Psychiat. Nervenkr. 179, 132.

Malamud, W., and Lowenberg, K. (1929). Arch. Neurol. Psychiat., Chicago, 21, 805

Meyer, J. E. (1949). Arch. Psychiat. Nervenkr., 182, 731.

Sjögren, T. (1931). Hereditas, Lund., 14. 197.

Sjövall, E., and Ericsson, E. (1933). Acta path. microbiol. scant., Suppl. 16, 460.

Spielmeyer, W. (1905). Nissls Beitr., 2, H, 1. Cited by Josephy.

Stengel, E. (1826). Eyr et medicinsk Tidsskrift, Christiania, 1, 347.

Vogt, H. (1905). Mschr. Psychiat., Neurol., 18, 161, 310.' Cited by Josephy. 